\title{
ARVEJAS (Pisum sativum) DE VAINA COMESTIBLE "SUGAR SNAP": ANTECEDENTES Y COMPORTAMIENTO EN EL SUR DE CHILE
}

\author{
Edible-podded sugar snap peas (Pisum sativum): description and behavior \\ in southern Chile
}

\author{
Mario Mera ${ }^{1}$ *, Elizabeth Kehr'1, Jaime Mejías', Mónica Ihl², y Valerio Bifani²
}

\begin{abstract}
A B S T R A C T
Sugar snap peas (Pisum sativum L.) are edible-podded peas that, unlike snow (Chinese) peas, have thick pod walls. They are the result of the combination of at least six recessive genes controlling pod and grain traits, and two others are being incorporated in modern cultivars. Sugar snap peas may be consumed fresh or frozen, and are highly appreciated in the United States and other countries. Six genotypes from the USA were evaluated at two locations in La Araucanía Region in southern Chile in 2004 and 2005. Most sugar snap varieties showed yields similar to a freezable cultivar used as a check. Yield differences among sugar snap varieties were small and inconsistent; however, cv. Sweet Ann performed relatively well and showed the best freezing quality. Over $15 \mathrm{t} \mathrm{ha}^{-1}$ of pods were obtained under favorable conditions, suggesting that yields around $10 \mathrm{t} \mathrm{ha}^{-1}$ are quite achievable commercially. All varieties presented, at variable levels, suture on both dorsal and ventral sides of the pods. A mild frost $\left(-2{ }^{\circ} \mathrm{C}\right)$, harmless to the plant, may also deteriorate the appearance of the pods. Sugar snap peas are a delicate produce and, if well managed, can be a sound option to diversify Chilean horticultural offer.
\end{abstract}

Key words: edible pod, horticultural pea, freezing vegetable.

\section{R E S U M E N}

Las arvejas (Pisum sativum L.) "sugar snap" son el resultado de la combinación de al menos seis genes recesivos que controlan características de la vaina y el grano, y otros dos genes se están sumando en variedades modernas. Es un tipo de vaina comestible que, a diferencia de las "sinhilas", tiene vainas de paredes engrosadas. Pueden consumirse en estado fresco o congelado y han tenido gran aceptación en Norteamérica y otros países. Seis genotipos provenientes de los Estados Unidos fueron evaluados en dos localidades de la Región de La Araucanía, Chile, durante 2004 y 2005 . La mayoría de las variedades "sugar snap" dieron rendimientos similares a un cultivar para congelado utilizado como testigo. Las diferencias de rendimiento entre las variedades "sugar snap" fueron pequeñas y no consistentes, sin embargo, la variedad Sweet Ann se comportó relativamente bien y mostró la mejor aptitud para congelado. Se obtuvieron rendimientos superiores a $15 \mathrm{tha}^{-1}$ de vaina bajo condiciones ambientales favorables, sugiriendo que es posible obtener $10 \mathrm{tha}^{-1}$ de vaina en producciones comerciales. Las vainas de las variedades evaluadas presentaron, en magnitud variable, haces de fibra en las suturas dorsal y ventral, que deben ser retirados antes del consumo. Una helada suave (alrededor de $-2{ }^{\circ} \mathrm{C}$ ), inofensiva para las plantas, puede también deteriorar la apariencia de las vainas. Las arvejas "sugar snap" son un producto delicado, y con un buen manejo podrían diversificar la oferta hortícola del país.

Palabras clave: vaina comestible, arveja hortícola, hortaliza congelable.

\footnotetext{
${ }^{1}$ Instituto de Investigaciones Agropecuarias, Centro Regional de Investigación Carillanca, Casilla 58-D, Temuco, Chile.

E-mail:mmera@inia.cl *Autor para correspondencia.

${ }^{2}$ Universidad de La Frontera, Departamento de Ingeniería Química, Casilla 54-D, Temuco, Chile.

Recibido: 30 de noviembre de 2006. Aceptado: 20 de marzo de 2007.
} 


\section{INTRODUCCIÓN}

El consumo de hortalizas en Chile ha aumentado sostenidamente durante las últimas décadas, particularmente las procesadas. Actualmente, más del $90 \%$ de los productos congelados en Chile son hortalizas. Entre los elementos vigorizantes del mercado de congelados se encuentran, una mayor demanda por productos de fácil y rápida preparación, una menor posibilidad de conseguir ayuda para labores del hogar, una mayor seguridad sanitaria asociada al consumo de alimentos congelados, la introducción masiva del horno microondas, una tendencia a la mayor incorporación de alimentos de origen vegetal en la dieta, y una mayor receptividad del consumidor chileno a productos novedosos, especialmente cuando son bien presentados. Este conjunto de elementos brinda un escenario propicio para la introducción de nuevos productos aptos para el congelamiento en Chile.

Un tipo de arveja desconocido en Chile es el llamado "sugar snap", que ha tenido gran aceptación en Norteamérica y está siendo importante en Europa. Este tipo puede ser consumido fresco y también congelado. Podría ser bien acogido en nuestro país, ampliando la oferta hortícola actual. Como ocurre en Norteamérica, podría ser adoptado para procesamiento por la industria del congelado, y en extensiones menores, por pequeños agricultores, con destino al mercado fresco. Incluso, puede ser un producto exportable en fresco para el mercado de Estados Unidos y Europa.

Un primer paso para conocer la opción de este nuevo tipo en nuestro país, es evaluar la adaptación de los materiales disponibles a nuestras condiciones de cultivo, dando énfasis a la introducción de materiales recientes, que incorporan mejoras tales como menor altura, concentración de madurez, mayor resistencia a tendedura temprana y resistencia a algunas enfermedades. Este artículo tuvo por objetivo entregar antecedentes sobre este nuevo tipo de arveja, así como resultados de su evaluación en la Región de La Araucanía.

\section{ANTECEDENTES}

Las arvejas "sugar snap" se caracterizan por poseer vainas dulces de paredes suculentas. Se han descrito arvejas semejantes en la literatura desde fines de 1800, con diferentes nombres (Myers et al., 2001). La primera variedad comercial del tipo "sugar snap" fue obtenida por el Dr. Calvin Lamborn en la empresa Gallatin Valley Seed (Thorndike, 1983) y se dio a conocer en Estados Unidos a principios de los años 80. Así como ocurre en el tipo de vaina comestible conocido en Chile como arvejas "sinhilas" ("snow peas", "China o Chinese peas" para el mercado norteamericano), la legumbre de las arvejas "sugar snap" carece de pergamino, la película fibrosa que recubre interiormente las paredes de la vaina. La ausencia de esta película fibrosa se debe a los genes recesivos complementarios, pero independientes, $p$ y $v$ (Blixt, 1974). No obstante, sólo algunas variedades del tipo "sugar snap" carecen del haz fibroso de las suturas ventral y dorsal de la vaina, cuya ausencia se ha atribuido al gen sin-2 en condición homocigótica recesiva (McGee y Bagget, 1992). El notable engrosamiento de las paredes de la vaina se debe al gen recesivo $n$ (Wehner y Gritton, 1981). En esencia, éste es el gen que diferencia a las "sugar snap" de las "sinhilas". Infortunadamente, tanto la presencia de $n$ como $\sin -2$ se ha asociado a un menor rendimiento; en el caso de $n$, debido a una reducción del tamaño y peso de la vaina (Wehner y Gritton, 1981), y en el caso de $\sin$-2, por efectos pleiotrópicos que reducen el tamaño de la planta y la vaina, particularmente bajo estrés (Myers et al., 2001).

Las variedades comerciales de arvejas "sugar snap" se caracterizan porque sus semillas son rugosas, de cotiledones verdes y ausencia de pigmentación antociánica. La diferencia entre la semilla rugosa y la lisa es controlada por un solo gen, donde el alelo recesivo $r$ produce una enzima defectuosa que retarda la transformación de azúcar a almidón (Myers et al., 2001). Como resultado, las semillas homocigotas recesivas son rugosas y con mayor contenido de azúcar. Las arvejas "sugar snap" requieren esta característica ya que se consumen con granos más desarrollados, en un estado de madurez más avanzado que las "sinhilas", que se consumen con granos muy pequeños. Por lo mismo, muchas variedades de arvejas "sinhilas" son de semilla lisa.

El color verde de los cotiledones, que confiere el alelo recesivo $i$, se prefiere al amarillo, debido a que al estado óptimo de cosecha la apariencia del cotiledón verde es más atractiva (Myers et al., 2001). La ausencia de antocianina en toda la planta (incluidas flores, vainas y cubierta seminal) es controlada por el gen recesivo $a$, que es importante 
puesto que las vainas con antocianina pueden presentar un leve sabor amargo. Las variedades recientes están sumando a todo lo anterior el follaje áfilo, donde los folíolos son transformados en zarcillos por acción del alelo recesivo af. Esta modificación confiere menor susceptibilidad a la tendedura temprana y mejor aireación del follaje (Ali et al., 1993).

Aunque las plantas de variedades modernas de arveja "sugar snap" son de baja altura, las primeras variedades, como 'Sugar Snap', pueden alcanzar hasta los $2 \mathrm{~m}$. No obstante, son utilizadas con elementos de sostén por pequeños horticultores en Norteamérica y Australia (Burt, 1999). Las bajas temperaturas no causan daño siempre que ocurran previo a la floración (Pleasant, 1995). Incluso heladas breves cercanas a $0^{\circ} \mathrm{C}$ generalmente son inocuas, aunque algunos genotipos pueden ser más susceptibles (Makasheva, 1983). Sin embargo, las heladas causan caída de flores y pueden afectar el aspecto de las vainas. Dado que el producto que se consume es la vaina entera, la apariencia de las mismas es un factor fundamental para la comercialización de las arvejas "sugar snap". Accidentes como heladas, que no causan daño a las plantas, pueden ocasionar sectores amarillentos sobre las vainas. Asimismo, temperaturas bajas durante la formación de vainas pueden causar curvatura de las mismas (Burt, 1999), aspecto que es indeseable.

A diferencia de las arvejas "sinhilas", que se cosechan cuando los granos están empezando a producir protuberancias en la vaina, el momento óptimo de cosecha de las arvejas "sugar snap" es en un estado más avanzado de madurez, cuando los granos están bien desarrollados y presionan las paredes de la vaina (Myers et al., 2001).

Las arvejas "sugar snap" se comercializan tanto al estado fresco como congeladas, sin embargo, no mantienen un aspecto atractivo luego del proceso de enlatado y por tanto no son utilizados en conservería (Myers et al., 2001). Como muchos productos hortícolas, tienen un período de poscosecha relativamente corto, sin embargo, pueden ser mantenidas frescas a baja temperatura por un par de semanas. La translocación de nutrientes desde las paredes de la vaina hacia el grano, durante el almacenamiento, disminuye la materia seca y el azúcar en las paredes, pero aumenta ambas en los granos (Basterrechea y Hicks, 1991). La técnica para el óptimo congelado industrial de las arvejas "sugar snap" ha sido mantenida en secreto (Anónimo, 1988a, 1988b). Sin embargo, pueden ser congeladas en el hogar y conservadas por largo tiempo si se escaldan en agua hirviendo por 2-3 minutos, se enfrían rápidamente con agua fría, y se drenan antes de ponerlas en una bolsa plástica y congelarlas (Anónimo, 1999). Las hortalizas requieren de un corto tratamiento térmico o escaldado para inactivar las enzimas, como proceso previo a la congelación y almacenamiento. De particular interés es la inactivación de la peroxidasa, la enzima más termorresistente dentro del grupo que causa deterioro de calidad en hortalizas mínimamente procesadas. Esta inactivación depende del tiempo y temperatura de escaldado; en algunos casos se ha encontrado una reactivación tras el almacenamiento bajo congelación (Garrote et al., 1984; Conejeros, 1999; Bifani et al., 2002; Albornoz, 2005).

\section{MATERIALES Y MÉTODOS}

Los materiales evaluados fueron las variedades Sugar Snap, Sugar Daddy, Sugar Prince, Sugar Lace II, SP-0895, y Sweet Ann (Cuadro 1). La semilla de Sugar Prince, Sugar Lace II, SP-0895 fue proporcionada por Syngenta y la semilla de Sweet Ann por Seminis. Estas empresas han realizado, considerando el trabajo previo de casas de semilla que han adquirido, la mayoría del mejoramiento genético del tipo "sugar snap". La variedad Mariner, para congelado, fue incluida como testigo. También se sembraron como testigos las variedades Snow Green y EP-9016, del tipo "snow", pero no se incluyen aquí los datos sino sólo comentarios comparativos. Los análisis de varianza y pruebas de comparación múltiple (Figuras 1 y 2) para ambas temporadas se realizaron con el programa JMP (SAS Institute, 2003).

\section{Temporada 2004-2005}

Los ensayos se establecieron el 9 y 11 agosto de 2004 en Carillanca ( $38^{\circ} 41^{\prime}$ lat. Sur, $72^{\circ} 25^{\prime}$ long. Oeste) y Maquehue ( $38^{\circ} 46^{\prime}$ lat. Sur, $72^{\circ} 41^{\prime}$ long. Oeste), respectivamente, localidades ubicadas 20 $\mathrm{km}$ al noreste y $5 \mathrm{~km}$ al suroeste de Temuco, respectivamente. Se prefirió la siembra a salidas de invierno para evitar enfermedades de incidencia invernal. El sector Maquehue se eligió porque concentra un grupo numeroso de pequeños horticultores que abastecen el mercado fresco de la ciudad de Temuco. Las parcelas consistieron en cinco surcos de $4 \mathrm{~m}$ de largo separados a $35 \mathrm{~cm}$ y se dispusieron 
Cuadro 1. Clasificación comercial, propietario actual, tipo de follaje, altura de planta, presencia o ausencia de sutura de la vaina según propietarios, y resistencia a enfermedades según propietarios, de seis variedades de arveja (Pisum sativum L.) tipo "sugar snap" y un testigo para congelado evaluados en Carillanca y Maquehue, Región de La Araucanía, Chile.

Table 1. Commercial classification, current owner, foliage type, plant height, presence or absence of strings in pods according to owners, and disease resistance according to owners, of six sugar snap pea (Pisum sativum $\mathrm{L}$.) cultivars and a freezing pea cultivar evaluated at Carillanca and Maquehue, La Araucanía Region, Chile.

\begin{tabular}{llllcll}
\hline Material & $\begin{array}{c}\text { Tipo } \\
\text { comercial }\end{array}$ & $\begin{array}{c}\text { Propietario } \\
\text { actual }\end{array}$ & Follaje & $\begin{array}{c}\text { Altura } \\
\text { planta } \\
\text { (cm) }\end{array}$ & Sutura & Resistencia a enfermedades \\
\hline Sweet Ann & Sugar snap & Seminis & Convencional & 80 & Ausente & Oídio y mildiú \\
Sugar Snap & Sugar snap & Syngenta & Convencional & 120 & Presente & Fusarium raza 1 \\
Sugar Prince & Sugar snap & Syngenta & Convencional & 81 & Ausente & Oídio \\
SP-0895 & Sugar snap & Syngenta & Áfilo & 77 & Ausente Oídio \\
Sugar Lace II & Sugar snap & Syngenta & Áfilo & 76 & Ausente Oídio y BLRV \\
Sugar Daddy & Sugar snap & Syngenta & Convencional & 82 & Ausente Oídio, tolerancia a BLRV \\
Mariner $(\mathrm{T})^{1}$ & Congelado & Rogers Brothers & Convencional & 76 & Presente & Fusarium raza 1, oídio, PEMV, PLRV \\
\hline
\end{tabular}

${ }^{1}$ T: testigo; BLRV: Virus del enrollamiento de la hoja del frejol (Bean leaf roll virus); PEMV: Virus del mosaico con excrescencia de la arveja (Pea enation mosaic virus); PLRV: Virus del enrollamiento de la hoja de la arveja (Pea leaf roll virus).

en un diseño de bloques completos aleatorizados con cuatro repeticiones. Se sembró a razón de 80 semillas $\mathrm{m}^{-2}$. Se fertilizó sólo con fósforo y potasio, de acuerdo a análisis de suelo, y se aplicó el herbicida simazina de preemergencia a razón de $1,0 \mathrm{~kg}$ $\mathrm{ha}^{-1}$ de ingrediente activo. Un sistema de cintas permitió regar por goteo.

Se realizaron muestreos por parcela para observar la nodulación en las raíces. La cosecha se realizó en una sola oportunidad. Como método para definir la madurez de las vainas comestibles se adoptó un criterio visual, considerando como momento óptimo de cosecha cuando los granos estuvieron bien desarrollados, y presionando las paredes de la vaina (Myers et al., 2001).

\section{Temporada 2005-2006}

Los ensayos se establecieron en dos épocas en cada localidad, 23 de agosto y 26 de septiembre de 2005 en Carillanca, y 2 y 27 de septiembre de 2005 en Maquehue. Las parcelas, el diseño experimental, el establecimiento y el manejo fueron similares a los de la temporada 2004-2005. La segunda época de cada localidad no contempló la variedad Sugar Lace II por escasez de semilla. Se determinó el primer nudo reproductivo, el número de nudos reproductivos, y el número de vainas por planta midiendo cinco plantas tomadas al azar por parcela. Con el fin de determinar el comportamiento para congelado, $30 \mathrm{~g}$ de arvejas frescas se escaldaron, totalmente sumergidas, en $3 \mathrm{~L}$ de agua en un baño termorregulado, a temperaturas de $80,85,90,95$, $98{ }^{\circ} \mathrm{C}$ con tiempos de $2,4,6$ y 8 min en cada temperatura. Terminado el escaldado se enfriaron rápidamente en abundante agua con hielo. Para cada tiempo y temperatura se analizó en triplicado la actividad residual de peroxidasa en forma cualitativa. Para ello se aplicó 3 gotas de $\mathrm{H}_{2} \mathrm{O}_{2}$ y 2 gotas de guayacol, suficiente para cubrir pincelando la mitad de la arveja; se dejó 1 min y se fotografió. Luego se calculó el porcentaje de actividad de peroxidasa en función de la superficie que presentó cambio de color. De la pendiente de la recta que se obtiene al graficar, para cada temperatura, el logaritmo de la actividad enzimática en función del tiempo, se obtiene el valor del parámetro cinético $\mathrm{D}$, que indica el tiempo requerido a cada temperatura para reducir en un $90 \%$ la actividad enzimática (Bifani et al., 2002; Albornoz, 2005).

\section{RESULTADOS Y DISCUSIÓN}

\section{Desarrollo del cultivo}

Se obtuvieron poblaciones que fluctuaron generalmente entre 60 y 70 plantas $\mathrm{m}^{-2}$. Tal densidad permitió alcanzar una buena cobertura de suelo alrededor de 75 días después de la siembra y suprimir el desarrollo de malezas. Esto es importante debido a la escasez de herbicidas de aplicación posemergente para controlar malezas de hoja ancha en arveja. 
En 2004, el desarrollo de las plantas y la intensidad del color verde del follaje se apreciaron bajo lo normal en Carillanca, posiblemente debido al efecto residual de herbicidas aplicados en temporadas previas. Este efecto no correspondió a la fitoxicidad por simazina, que causa clorosis en los márgenes de los folíolos y se presenta en estados tempranos de desarrollo. Por tanto, se considera que los materiales evaluados toleraron bien el herbicida simazina de preemergencia en dosis usual para arveja. En Maquehue el desarrollo de las plantas fue excelente, sin embargo, las frecuentes lluvias de la temporada promovieron el ataque de hongos. Como es usual en la zona sur, se observó el complejo Ascochyta y Pseudomonas syringae (Hagedorn, 1984), con la irregularidad espacial que los caracteriza. En general, los materiales evaluados mostraron mayor susceptibilidad a estas enfermedades que las variedades modernas de arveja proteaginosa. No se presentó oídio (Erysiphe pisi) durante las temporadas de evaluación, enfermedad que puede constituir un problema en siembras tardías. De acuerdo a Syngenta, 'SP-0895', 'Sugar Lace II' y 'Sugar Prince' son resistentes a oídio (Gritton y Myers, 2005), lo cual permitiría atrasar la siembra a fin de evitar un ambiente húmedo. De acuerdo a Seminis, 'Sweet Ann' es resistente a oídio y también a mildiú (Peronospora viciae) (Gritton y Myers, 2005).

En 2005, el desarrollo alcanzado por las plantas en los ensayos de Carillanca fue normal. En Maquehue hubo menor emergencia en la primera época de siembra, y las plantas alcanzaron un menor desarrollo respecto a la segunda época de siembra. Esto se atribuyó a que la preparación de suelos se realizó con demasiada humedad, perdiéndose estructura. Cabe señalar que la intención era sembrar la primera época durante agosto, pero las lluvias de la temporada no lo permitieron. Sólo se logró sembrar la primera época a inicios de septiembre, con el suelo en condiciones que probablemente no hubieran afectado a un cereal, pero que sí perjudicaron a la arveja.

La floración se inició la última semana de octubre en Maquehue y la primera semana de noviembre en Carillanca. Los días de siembra a floración y la correspondiente sumatoria de grados días se indican en el Cuadro 2.

La tendedura se presentó más temprano en las variedades de follaje convencional y particularmente en la variedad Sugar Snap, de mayor altura. La tendedura se retardó, y fue menos severa, en los materiales de follaje áfilo. En ambas localidades se realizó la cosecha la última semana de diciembre. La excesiva altura de planta de la variedad Sugar Snap (Cuadro 1), la primera de este tipo, es una característica indeseable. Sin embargo, esta variedad puede producir bien con estructuras de sostén, en pequeñas superficies y con una cosecha parcializada. El resto de los materiales tiene una altura agronómicamente conveniente (Cuadro 1), con mayor concentración de la madurez.

\section{Rendimiento}

En 2004, el rendimiento en ambas localidades fue bajo, en particular si se considera que los ensayos

Cuadro 2. Días a inicio de floración y grados días base $4,4^{\circ} \mathrm{C}$ desde siembra a inicio de floración y sólidos solubles de seis variedades de arveja (Pisum sativum $\mathbf{L}$.) tipo "sugar snap" y un testigo para congelado, evaluados en 2004 en Carillanca y Maquehue, Región de La Araucanía.

Table 2. Initiation of flowering, degree days over $4.4^{\circ} \mathrm{C}$ from sowing to flowering initiation, and soluble solids of six sugar snap pea (Pisum sativum $\mathrm{L}$.) cultivars and a freezing pea cultivar evaluated at Carillanca and Maquehue, La Araucanía Region.

\begin{tabular}{|c|c|c|c|c|c|c|}
\hline \multirow[t]{2}{*}{ Material } & \multicolumn{2}{|c|}{ Inicio de floración (d) } & \multicolumn{2}{|c|}{ Grados días $\left({ }^{\circ} \mathrm{C}\right)$ a inicio de floración } & \multicolumn{2}{|c|}{ Sólidos solubles ( $\left.{ }^{\circ} \mathrm{Brix}\right)$} \\
\hline & Carillanca & Maquehue & Carillanca & Maquehue & Carillanca & Maquehue \\
\hline Sweet Ann & 88 & 79 & 489 & 508 & 13,0 & 14,5 \\
\hline Sugar Snap & 88 & 79 & 489 & 508 & 11,6 & 12,8 \\
\hline Sugar Prince & 85 & 79 & 464 & 508 & 12,6 & 13,5 \\
\hline SP-0895 & 88 & 84 & 489 & 541 & 12,2 & 12,2 \\
\hline Sugar Lace II & 88 & 79 & 489 & 508 & 12,2 & 13,6 \\
\hline Sugar Daddy & 88 & 79 & 489 & 508 & 12,2 & 12,6 \\
\hline Mariner $(T)^{1}$ & 85 & 79 & 464 & 508 & - & - \\
\hline
\end{tabular}

${ }^{1} \mathrm{~T}$ : testigo. 


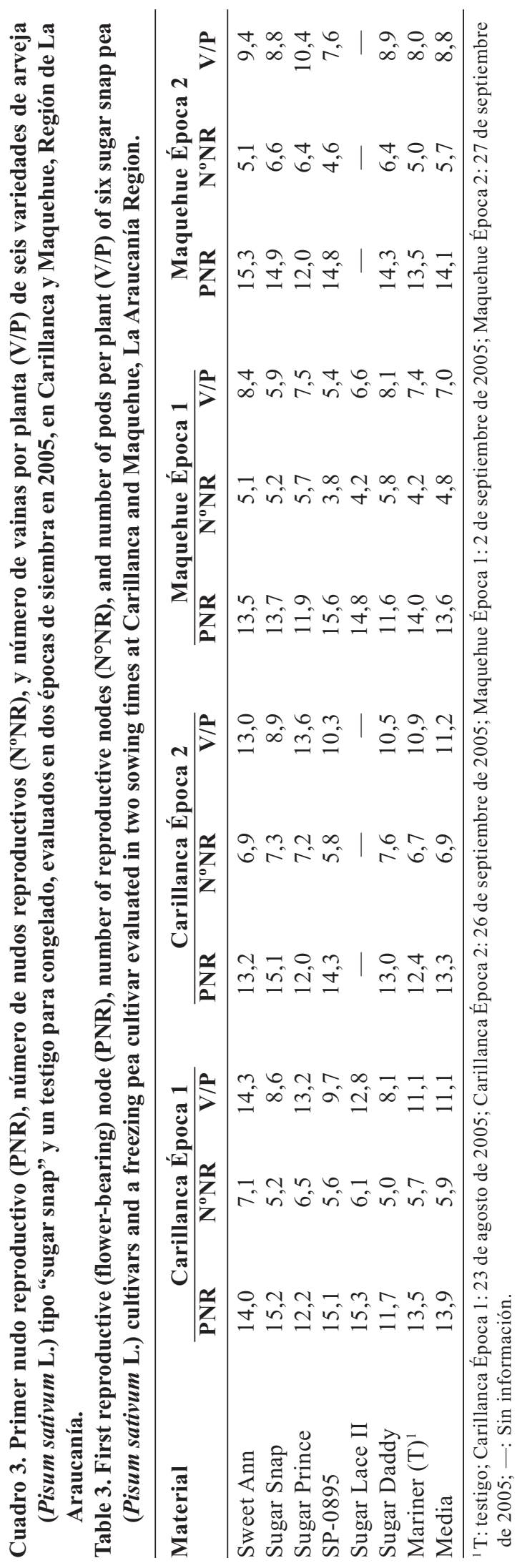

en La Araucanía con arvejas para congelado (como 'Mariner') frecuentemente rinden sobre $20 \mathrm{t} \mathrm{ha}^{-1} \mathrm{y}$ ocasionalmente sobre $30 \mathrm{t} \mathrm{ha}^{-1}$ de vaina verde por hectárea (Mera et al., 1999). Las diferencias significativas entre variedades fueron escasas y no consistentes (Figura 1). En ambas localidades se observó nodulación adecuada en todas las variedades, lo cual permite asumir que el rendimiento no estuvo limitado por la disponibilidad de nitrógeno.

En 2005 los rendimientos fueron mejores, particularmente en el caso de la segunda época de siembra (Figura 2). En Carillanca, la diferencia de rendimiento entre las fechas de siembra fue menor, no así en Maquehue, donde los rendimientos fueron notablemente más bajos en la primera fecha de siembra que en la segunda, coincidente con un menor desarrollo general de las plantas y una reducción del número de nudos reproductivos (Cuadro 3). Como se ha dicho previamente, la pérdida de estructura producto de una preparación de suelo realizada con exceso de humedad, probablemente disminuyó la aireación. En ambas épocas de siembra en Carillanca, y en la segunda época de siembra en Maquehue, varios materiales alcanzaron rendimientos entre 15 y $20 \mathrm{t} \mathrm{ha}^{-1}$, semejantes a la variedad testigo Mariner (Figura 2), lo cual permite suponer que bajo condiciones adecuadas, algunas variedades "sugar snap" pueden alcanzar rendimientos similares al de variedades para congelado.

Otros ensayos realizados en Carillanca y Maquehue, no reportados aquí, sugieren que el rendimiento puede aumentar con siembras más tempranas (julio), sin embargo, por la incidencia de enfermedades y el ambiente más húmedo, se corre el riesgo de perder una alta proporción de la producción por deterioro de vainas. De lo observado puede inferirse que la mejor calidad podría obtenerse con siembras relativamente tardías (fines de septiembre), disponiendo de riego para aquellas temporadas en que se requiera.

\section{Aspectos de calidad}

El contenido de sólidos solubles, variable ligada al contenido de azúcar, se encontró en un rango de 11,6 a $14,5^{\circ}$ Brix para las arvejas "sugar snap" (Cuadro 2). Observaciones previas han dado valores de 14,7 y $18,6^{\circ}$ Brix para las variedades Sugar Snap y Sugar Daddy, respectivamente (Ihl et al., 2001). Las diferencias probablemente se deban a distintos estados de madurez de las vainas. Las arvejas "sugar 
(a) Carillanca

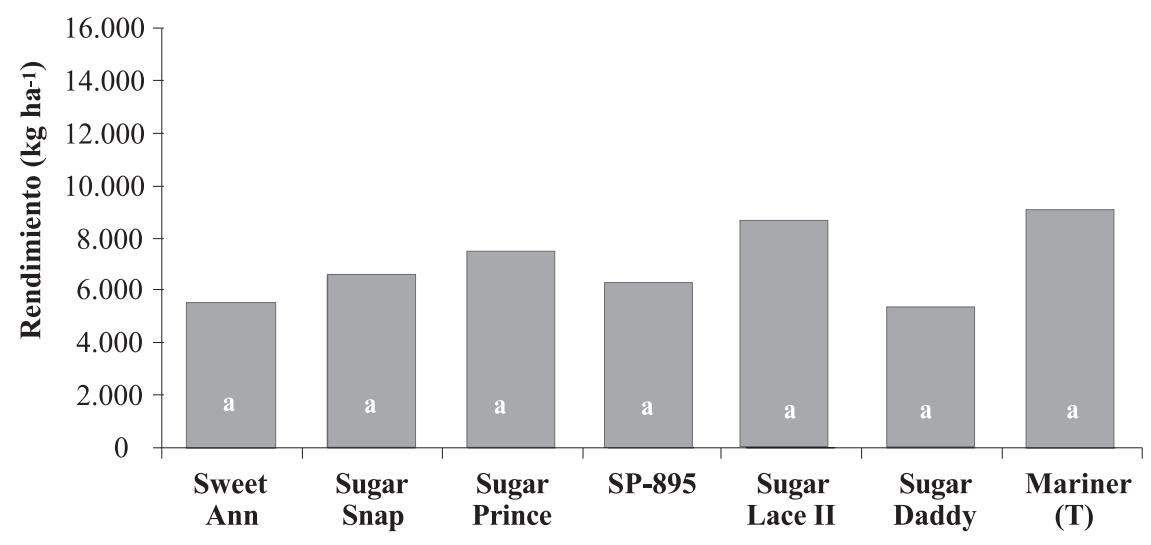

(b) Maquehue

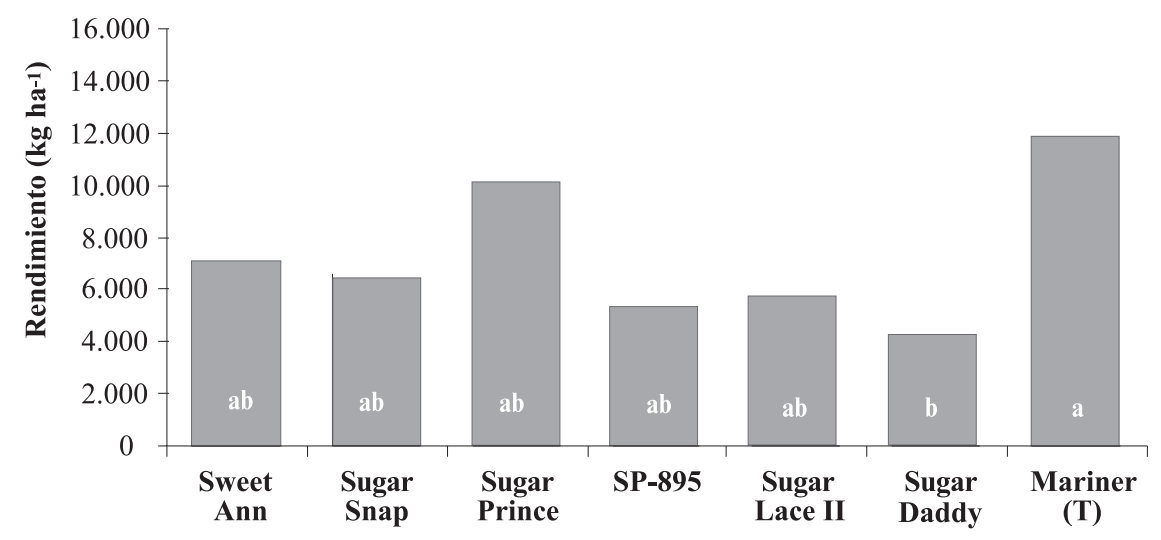

Figura 1. Rendimiento en vaina de variedades de arveja tipo "sugar snap" en dos épocas de siembra, durante 2004. (a) Carillanca, (b) Maquehue.

Figure 1. Yield (pods) of sugar snap cultivars in two sowing times in 2004. a) Carillanca, (b) Maquehue.

Letras diferentes indican diferencias significativas según Tukey $(P<0,05)$.

T: testigo.

snap" se cosechan en un estado de madurez en el cual los granos están cerca de alcanzar su tamaño máximo, y el período adecuado es más amplio que en el caso de arvejas para congelado, cuyo momento óptimo de cosecha puede ser determinado con un tenderómetro. El contenido de sólidos solubles reportado es claramente superior al de los porotos de vaina comestible, que se encuentra alrededor de 5 ${ }^{\circ}$ Brix, e inferior al de maíz dulce, con aproximadamente $19^{\circ}$ Brix (Nehring, 1985).

Bajo condiciones húmedas, como las que ocurrieron durante la primera temporada de evaluación, se comprobó que los pétalos pueden adherirse al extremo distal de algunas vainas y favorecer el de- sarrollo de hongos como Alternaria, Ascochyta y Penicillium (Dillard y Hunter, 1988). Lo anterior corrobora la información norteamericana (Anónimo, 1988a; 1988b), en el sentido que las actuales variedades de arveja "sugar snap" requieren un ambiente relativamente seco durante la formación de vainas para lograr una buena calidad.

Pese a que según sus propietarios varias variedades no presentan sutura de la vaina (Cuadro 1), en la presente evaluación todas presentaron hilos fibrosos en la sutura de las vainas. La presencia/ ausencia de fibra en las suturas de la vaina es difícil de establecer debido a que la expresión de este carácter es afectada por la temperatura. Sin embar- 

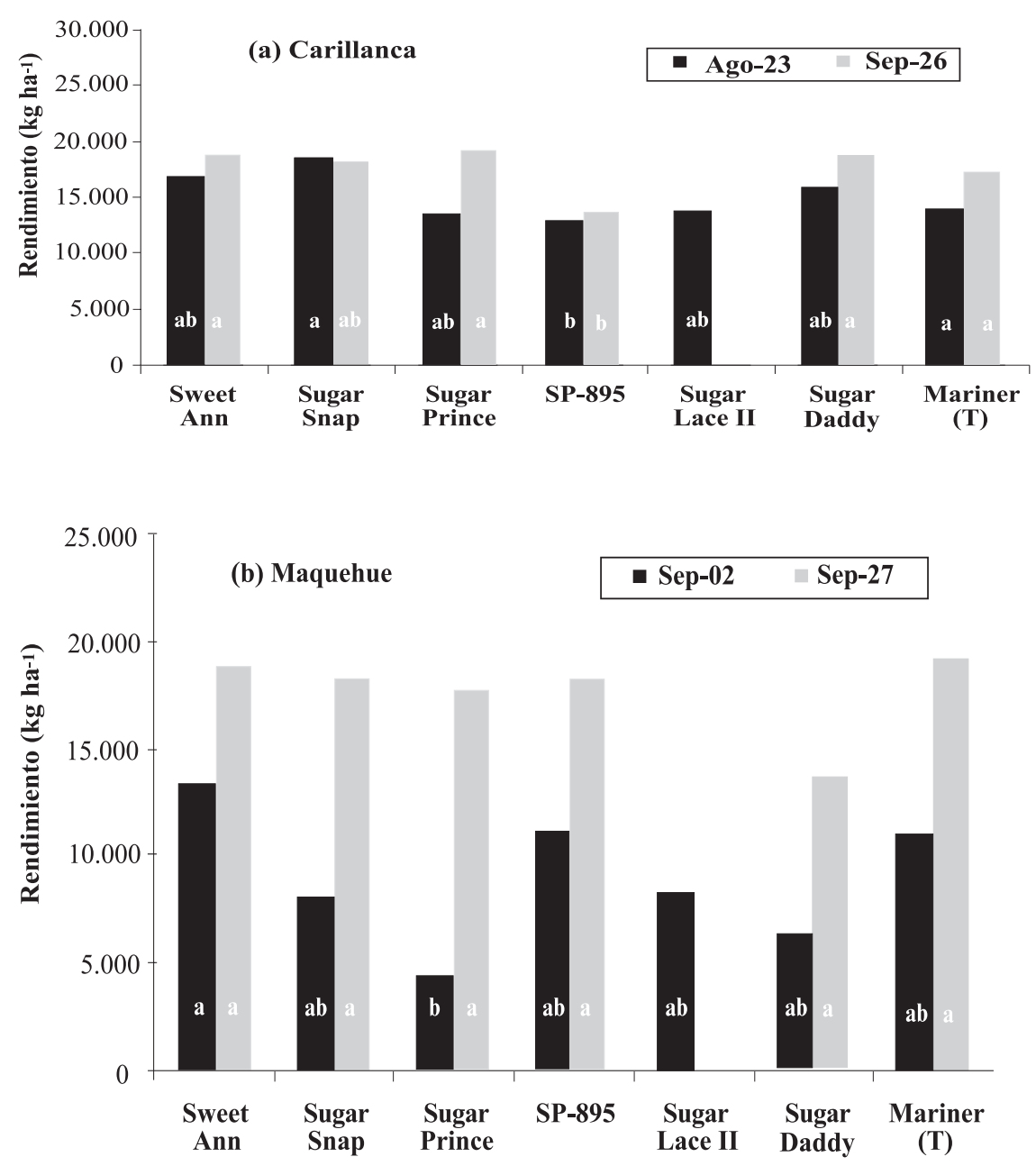

Figura 2. Rendimiento en vaina de variedades de arveja tipo "sugar snap" en dos épocas de siembra, durante 2005. (a) Carillanca, (b) Maquehue.

Figure 2. Yield (pods) of sugar snap cultivars in two sowing times in 2005. a) Carillanca, (b) Maquehue.

Letras diferentes dentro de una época de siembra indican diferencias significativas, según Tukey $(\mathrm{P}<0,05)$.

T: testigo.

go, en la variedad Sugar Daddy la fibra fue generalmente menor y cierta proporción de vainas careció de ella.

Las variedades de follaje áfilo (SP-0895 y Sugar Lace II) son más resistentes a la tendedura temprana y tienen buenas posibilidades de producir vainas limpias, que no entran en contacto con el suelo, pudiendo ser una opción adicional para el mercado fresco.

Las determinaciones en la Universidad de La Frontera, Temuco, han permitido estimar que almacenadas frescas a $5{ }^{\circ} \mathrm{C}$ las arvejas "sugar snap" mantie- nen buenas características de calidad durante 16 días (datos no mostrados). La variedad Sweet Ann obtuvo la mejor calificación en un panel de evaluación sensorial que consideró sabor, textura y turgencia.

\section{Comportamiento para congelado}

Los valores de reducción decimal $\mathrm{D}$, obtenidos de datos experimentales por regresión lineal, mostraron los mejores resultados a $95{ }^{\circ} \mathrm{C}$ (Cuadro 4). Para ambos sectores experimentales, la variedad Sweet Ann presentó el menor tiempo de inactivación de peroxidasa (1,69 min para Maquehue y 1,36 min para Carillanca). Al requerir un tratamiento térmico más corto para 
Cuadro 4. Tiempo de inactivación térmica de peroxidasa (D) de vainas de seis variedades de arveja tipo "sugar snap" y una del tipo "Chinese" (EP-9010), cultivadas en Carillanca y Maquehue, Región de La Araucanía.

Table 4. Thermal inactivation time for peroxidase (D) of pods from six sugar snap and one "Chinese" (EP-9010) pea cultivars grown at Carillanca and Maquehue, La Araucanía Region.

\begin{tabular}{lccccccc}
\hline \multirow{2}{*}{$\begin{array}{l}\text { Blanching } \\
\text { temperature } \\
\left({ }^{\circ} \mathbf{C}\right)\end{array}$} & \multicolumn{7}{c}{ Carillanca } \\
\cline { 2 - 8 } & S. Prince & Sweet Ann & S. Daddy & S. Snap & SP-895 & S. Lace II & EP-9010 \\
\hline $\mathbf{8 0}$ & 7,11 & 6,65 & 6,09 & 7,71 & 6,53 & 7,28 & - \\
$\mathbf{8 5}$ & 6,54 & 5,76 & 5,22 & 6,94 & 6,47 & 6,31 & - \\
$\mathbf{9 0}$ & 4,89 & 3,84 & 4,87 & 4,85 & 4,23 & 4,75 & 6,29 \\
$\mathbf{9 5}$ & 2,63 & 1,36 & 2,47 & 2,73 & 1,99 & 2,47 & 4,71 \\
$\mathbf{9 8}$ & 2,38 & - & 2,61 & - & 2,37 & - & 4,62 \\
\hline
\end{tabular}

\begin{tabular}{lccccccc}
\hline $\begin{array}{l}\text { Blanching } \\
\text { temperature } \\
\left({ }^{\circ} \mathbf{C}\right)\end{array}$ & \multicolumn{7}{c}{ Maquehue } \\
\cline { 2 - 7 } & S. Prince & Sweet Ann & S. Daddy & S. Snap & SP-895 & S. Lace II & EP-9010 \\
\hline $\mathbf{8 0}$ & 8,01 & 5,92 & 6,76 & 8,39 & 6,79 & 7,28 & 8,76 \\
$\mathbf{8 5}$ & 6,81 & 5,11 & 5,99 & 6,69 & 6,09 & 6,58 & 7,85 \\
$\mathbf{9 0}$ & 4,99 & 3,01 & 3,99 & 4,19 & 5,09 & 4,32 & 4,85 \\
$\mathbf{9 5}$ & 2,80 & 1,69 & 2,29 & 2,43 & 2,36 & 2,25 & 2,05 \\
$\mathbf{9 8}$ & 2,28 & - & 2,38 & - & 2,09 & - & 2,56 \\
\hline
\end{tabular}

inactivar la enzima se traduce en un producto con mejor color y mayor valor nutricional (Albornoz, 2005). No se apreció diferencias importantes para la inactivación enzimática de una misma variedad en los dos sectores de cultivo.

Como era de esperar, el menor tiempo de inactivación se obtuvo con la mayor temperatura de escaldado. Los valores obtenidos concuerdan con los reportados por Conejeros (1999), para $95^{\circ} \mathrm{C}$ con las variedades Sugar Snap $(2,48 \mathrm{~min})$ y Sugar Daddy (2,68 min). Valores similares han sido informados para soya con vaina, con $\mathrm{D}=1,14 \mathrm{~min}$ para $100^{\circ} \mathrm{C}$ (Sheu y Chen, 1991); acelga, con $\mathrm{D}=$ $0,50 \mathrm{~min}$ (Garrote et al., 1984), y poroto verde cv. Win, con $\mathrm{D}=0,94$ min para $95{ }^{\circ} \mathrm{C}$ (Bifani et al., 2002).

\section{Producción de semilla}

La producción de semilla de buena calidad es dificultada por las gruesas paredes de las vainas, que retienen la humedad y dilatan el secado del grano. Un porcentaje relativamente alto del grano cosechado adquiere un aspecto "oxidado", indicativo de calidad de semilla pobre.

\section{CONCLUSIONES}

Las variedades del tipo "sugar snap" evaluadas mostraron una buena adaptación a siembras de primavera en la zona sur de Chile. De acuerdo a los resultados, pueden obtenerse sin mayor dificultad rendimientos de $10 \mathrm{tha}^{-1}$ de vaina, sin embargo, es posible superar las $15 \mathrm{t} \mathrm{ha}^{-1}$ con condiciones favorables. Las siembras primaverales permiten escapar de varias enfermedades. Aunque el rendimiento relativo de las variedades no fue consistente, la variedad Sweet Ann resulta la más recomendable por combinar un buen rendimiento promedio con la mejor aptitud para congelado.

\section{RECONOCIMIENTOS}

La información presentada fue generada en el marco del proyecto FIA-PI-C-2003-3-A-015, financiado por la Fundación para la Innovación Agraria (FIA). Las empresas Seminis y Syngenta proporcionaron la semilla de las variedades indicadas. 


\section{LITERATURA CITADA}

Albornoz, E.K. 2005. Selección de siete variedades de arvejas de vaina suculenta comestible producidas en dos sectores de la IX Región, para consumo fresco y procesamiento agroindustrial. $276 \mathrm{p}$. Tesis Ingeniero en Alimentos. Universidad de La Frontera, Temuco, Chile.

Ali, S.M., B. Sharma, and M.J. Ambrose. 1993. Current status and future strategy in breeding pea to improve resistance to biotic and abiotic stresses. Euphytica 73:115-126.

Anonymous. 1988a. Sugar Snap Peas: The genetic engineering of a new gourmet vegetable. Food Prod. Mgmt. February 1988. p. 18-19, 30-31.

Anonymous. 1988b. Sugar Snap Peas - A sweet surprise. American Vegetable Grower. May 1988. p. 22-26.

Anonymous. 1999. Freezing snow peas and sugar snap peas. Preserving Food Safely - 01600794. Michigan State University Extension, East Lansing, Michigan, USA. Available at http://www.msue.msu.edu/msue/ imp/mod01/01600794.html Accessed 11 December 2003.

Basterrechea, M., and J.R. Hicks. 1991. Effect of maturity on carbohydrate changes in sugar snap pea pods during storage. Scientia Hort. 48:1-8.

Bifani, V., J. Inostroza, M.J. Cabezas, y M. Ihl. 2002. Determinación de parámetros cinéticos de peroxidasa y clorofila en judías verdes (Phaseolus vulgaris cv. Win) y estabilidad del producto congelado. Afinidad 497:57-64.

Blixt, S. 1974. The pea. Vol. 2. p. 181-221. In R.C. King (ed.). Handbook of genetics. Plenum Press, New York, USA.

Burt, J. 1999. Growing snow peas and sugar snaps in Western Australia. Farmnote No50/99. Agriculture Western Australia, South Perth, Australia.

Conejeros, E.M. 1999. Determinación de calidad y actividad enzimática en porotos verdes (Phaseolus vulgaris cv. Win) escaldados para distintos métodos de congelación. 153 p. Tesis Ingeniero en Alimentos. Universidad de La Frontera, Temuco, Chile.

Dillard, H.R., and J.E. Hunter. 1988. Fungal-induced blemishes of snap peas associated with adherence of blossom parts to the pods. HortScience 23:847-848.
Garrote, R., R. Bertone, E. Silva, y O. Sbodio. 1984. Inactivación térmica de las enzimas polifenoloxidasa y peroxidasa en acelga (Beta vulgaris cicla). Archivos Latinoamericanos de Nutrición 34:199-207.

Gritton, E.T., and J.R. Myers. 2005. Vegetable cultivar descriptions for North America. Pea-Green (M-Z), Lists 1-26 Combined. Available at http://cuke. hort.ncsu.edu/cucurbit/wehner/vegcult/peagreenmz. html Accessed 13 July 2007.

Hagedorn, D.J. 1984. Compendium of pea diseases. 57 p. Am. Phytopathol. Soc., St. Paul, Minnesota, USA.

Ihl, M., E. Conejeros, M. Mera, and V. Bifani. 2001. Quality changes of two sugar snap pea cultivars during cold storage. Acta Hortic. 553:749-751.

Makasheva, R.K. 1983. The pea. 267 p. Translated from Russian by B.R. Sharma. Oxonian Press, New Delhi, India.

McGee, R.J., and J.R. Bagget. 1992. Inheritance of stringless pod in Pisum sativum L. J. Am. Soc. Hortic. Sci. 117:628-632.

Mera, M., J.M. Alcalde, y S. Ferrada. 1999. Arvejas para congelado en el sur: Sorprendente potencial de rendimiento. Tierra Adentro 28:23-25.

Myers, J.R., J.R. Bagget, and C. Lamborn. 2001. Origin, history, and genetic improvement of the snap pea (Pisum sativum L.). Plant Breed. Rev. 21:93-138.

Nehring, P. 1985. Frischgemüse. p. 311-361. In J. Schormüller (ed.). Handbuch der Lebensmittelchemie. Springer Verlag, Berlin, Deutschland.

Pleasant, B. 1995. These peas are a snap. Organic Gardening. January 1995. p. 66-70.

SAS Institute. 2003. JMP Statistical Discovery Software. SAS Institute Inc., Cary, North Carolina, USA.

Sheu, S. and A. Shen. 1991. Lipoxygenase as blanching index for frozen vegetables soybeans. J. Food Sci. 56:448-451.

Thorndike, J. 1983. The making of the 'Sugar Snap' pea. Horticulture 61:14-23.

Wehner, T.C., and E.T. Gritton. 1981. Effect of the $n$ gene on pea pod characteristics. J. Am. Soc. Hortic. Sci. 106:181-183. 